\title{
The Piéron function in the threshold region
}

\author{
DELPHINE PINS and CLAUDE BONNET \\ Université Louis Pasteur, Strasbourg, France
}

\begin{abstract}
The Piéron function (Piéron, 1914, 1920, 1952) describes the decay of reaction time (RT) when the intensity of the stimulus is increased. It is generally demonstrated within a suprathreshold range of intensities. However, in some studies, for the lowest range of intensities, the exponent of the function is clearly greater than that for the upper ranges of intensities. Such an increase in the exponent for the lowest intensities is assumed to result from a combined effect of stimulus intensity and of stimulis uncertainty in detection. Our first experiment used luminance levels that covered all the scotopic range and a spatial two-alternative forced-choice task in which both accuracy and RT were measured. It demonstrated a drastic increase in the exponent in the Piéron function when the intensities reached the threshold region. Since the estimates of the threshold region may have been biased by the use of a much larger range of luminances, a second experiment was conducted using luminances that covered only the threshold region. This experiment confirmed the previous estimates for the threshold region.
\end{abstract}

Since Exner (1868), Wundt (1874), Cattell (1886), and Piéron (1914), many studies in different sensory modalities have demonstrated an inverse relationship between simple reaction time (SRT) and stimulus intensity. A possible exception occurs when color stimuli has been used (Luce, 1986). Various equations have been suggested to summarize the data. Bujas (1935) proposed a rectangular hyperbola, which is a special case of the Pieron function (see Equation 1) for $\alpha=1$. This function was first adopted by Yamamoto and Kawamura (1981), but was later slightly modified by Yamamoto et al. (1982). Hara (1955) proposed a linear logarithm function that might have led to a change in Bujas's original function by adding logarithms. Woodworth and Schlosberg (1955) suggested an exponential function. Other functions, such as the symmetric logistic and Michaelis's functions (Schweickert, Dahn, \& McGuigan, 1988), have also been investigated. In a recent paper, Bonnet, Zamora, Buratti, and Guirao (1999) compared these different solutions using a large sample of RTs in several taste concentration experiments. Overall, the Piéron function provided the best fit to the data.

Piéron's law (Piéron, 1914, 1920, 1952) describes the decrease of simple reaction time (SRT) with the increase of supraliminal intensities of a given stimulus by a power function in the form:

$$
\left(\mathrm{RT}-t_{0}\right)=\beta I^{-\alpha},
$$

where RT is SRT, $t_{0}$ is the asymptotic RT reached at the highest stimulus intensities, $\beta$ is a free parameter, $I$ is the in-

The authors thank J. C. Baird and L. Allan. as well as, an anonymous reviewer for their helpful suggestions and comments on a previous version of this paper. Correspondence should be addressed to D. Pins, Laboratoire de Psychopathologie et Pharmacologie de la Cognition, INSERM U405, Clinique Psychiatrique, Hôpitaux Universitaires de Strasbourg, 1 Place de l'Hôpital, 67091 Strasbourg, France (e-mail: pins@gren.ucl.ac.be). tensity of the stimulus, and $\alpha$ is the exponent of the function. The functional significance of these parameters is still uncertain. However, parameters $\alpha$ and $t_{0}$ appear to be specific for a given sensory modality (see Bonnet, 1992a, 1992b). Parameter $t_{0}$ appears to represent the combination of at least two constant parameters: The duration of the motor component and a specific processing time for a given sensory modality. Because $t_{0}$ varies between sensory modalities, its functional significance is presumably more sensory than decisional (see Bonnet, 1992a). However, $t_{0}$ may be shown to vary with the task (see Pins \& Bonnet, 1996).

Although such an effect of stimulus intensity on RT has been questioned in choice reaction time (CRT) tasks when visual stimuli were used (e.g., Luce, 1986), some authors have shown that CRT decreases when the luminance of the stimulus increases (e.g., Lappin \& Disch, 1972; Pachella \& Fisher, 1969; Posner, 1986). Moreover, in experiments in which the subject had to localize whether a visual stimulus, which varied in luminance from trial to trial, appeared to the left or to the right of a fixation point, Pins and Bonnet (1996) have shown that the exponent of the Piéron function does not vary with the complexity of the psychophysical task. The effect of intensity, estimated by the value of the exponent $\alpha$, is much the same in CRT tasks as in SRT tasks. The exponent of the Piéron function does not change with the complexity of the additional stages that are required between luminance processing and the decision process. A change in RT as a function of luminance probably arises only as a consequence of the luminance processing stage.

Most RT models assume that responses result basically from some kind of accumulation of information over time (e.g., Grice, 1968; Link, 1978, 1992; Luce, 1986; Luce \& Green, 1972; McGill, 1961, 1963). When stimulus intensity increases, the rate of neural impulses increases (e.g., Bartlett \& Doty, 1974; Levick, 1973; Marrocco, 1975; Stone \& Fukuda, 1974; Tepas \& Armington, 1962). The rate of firing of neurons is the biological support of the assumed mechanism. The higher the intensity, the higher the 
rate of neural firing, and the faster the rate of accrual of selected information. A second factor that comes into play is the critical accumulation level at which the response is triggered. The first factor is the result of a sensory mechanism, the second is the result of the decisional process (Bonnet, 1996; Pins \& Bonnet, 1996). Assuming that the response criterion of the subject is constant, RT is inversely related to the rate of accrual of information (e.g., Link, 1992).

It has been pointed out (e.g., Bonnet, 1992a; Restle, 1961) that sometimes the fit of a single function over an entire dynamic range of intensities is optimized when the lowest intensities are discarded. For instance, Restle (1961) reanalyzed Chocholle's (1940) RTs for pure tones and concluded that two functions provided a better fit to the data than one. Moreover, the function fitted for the lowest range of intensities appears to have a much higher exponent than the one fitted for upper intensities. Similar conclusions can be reached from reanalyzing Piéron's data, as he expressed the intensities of his stimuli relative to the threshold. In fact, Baird (1997) and Stevens (1975) have mentioned that magnitude estimation data also yield a steeper exponent of the power function near threshold than they do in the suprathreshold range. This is a further argument for the assumption that the exponents in Stevens's and Piéron's functions should be the same (Baird, 1997; Norwich, 1993).

The question here is to determine the factor responsible for the drastic elevation of the exponent in the Piéron function in the lowest range of luminance intensities. The simplest assumption that can be put forward is that the exponent rises when the intensities approach the threshold region (i.e., when stimulus uncertainty increases), while uncertainty is known to lengthen the RT (e.g., Link, 1992). In the threshold region, uncertainty increases as intensity decreases. The RT, which increases when intensity decreases, should therefore increase even faster in the threshold region compared to in suprathreshold ranges.

The intensities used by Piéron (1914) and Chocholle (1940) started from threshold. Hence, presumably some of the lowest intensities remained in the threshold region. However, they did not explicitly mention this. Because Piéron and Chocolle used a SRT task, an RT could be obtained even when the target was missed by the subject. The question becomes whether or not the increase in the exponent is limited to the threshold region (i.e., the region in which errors are detection errors).

In the present experiments, a CRT task was used in order to estimate the probability of correct detection of the target together with the RT. In previous experiments (Pins \& Bonnet, 1996), the exponent of the Pierron function was not affected by the type of task (i.e., whether it involves SRT or CRT).

\section{EXPERIMENT 1 \\ Determining the Threshold Region in Scotopic Luminance Levels}

In this experiment, luminance levels covered the scotopic range, from a value just inferior to threshold to the beginning of the mesopic range (e.g., Pins \& Bonnet, 1997). The target was presented in a spatial two-alternative forced-choice (2AFC) paradigm. This experiment was run in order to test the assumption that, in the threshold region, there was a combined effect of luminance and stimulus uncertainty on processing time. Two Piéron functions were expected, one of them with a larger exponent for the range of intensities near threshold.

\section{Method}

Subjects. Four trained subjects took part in the experiment. One of them was the first author. The subjects had normal or correctedto-normal vision.

Procedure. The stimuli and experimental design were identical to those used by Pins and Bonnet (1996) in a localization experiment (Experiment 3). The stimuli were presented binocularly on a highresolution video monitor (Visionor Model M 5I CHR No. 1007, Lille, France). The stimuli were generated through a PC-compatible computer (HP20 0386) that used a special graphics adaptator (GALAXY ref. SA-1019A, Evroz, Tel Aviv) that provided a display of $1024 \times 768$ pixels at a $60-\mathrm{Hz}$ frame rate (noninterlaced). Careful calibration of each RGB combination was carried out with a homemade photomultiplier standardized with a Pritchard photomultiplier. The values were cross-checked with a CS 100 Minolta photometer.

The stimuli were luminous vertical rectangles $(30 \times 7.5 \mathrm{~min}$ of arc $)$ presented during $83 \mathrm{msec}$ on a dark background $\left(0.02 \mathrm{~cd} / \mathrm{m}^{2}\right)$ in a dark room. The subjects were placed at a viewing distance of $70 \mathrm{~cm}$. The head position was stabilized by means of a chinrest. They looked at a central fixation dot ( $1.5 \mathrm{~min}$ of arc diameter, $\left.10 \mathrm{~cd} / \mathrm{m}^{2}\right)$. In each trial, a luminous rectangle appeared, with its center located randomly at $1.25^{\circ}$ left or right from the fixation point. Ten scotopic luminance levels of the target were used. They followed an approximately geometrical series $(0.104,0.116,0.133,0.151,0.170,0.194,0.219,0.246$, 0.280 , and $0.314 \mathrm{~cd} / \mathrm{m}^{2}$ ). These luminance levels ranged from scotopic levels (values inferior to the detection threshold: $0.120 \mathrm{~cd} / \mathrm{m}^{2}$ ) to mesopic levels. The different luminance levels were presented in mixed blocks. Each session started with a darkness adaptation period.

The RT measurements were rounded to the closest millisecond as indicated by the external clock driven by the computer. The appearance of the target was preceded by an auditory warning signal $(1000 \mathrm{~Hz}, 500 \mathrm{msec})$. Five preparatory periods $(450,550,650,750$, and $850 \mathrm{msec}$ ) were used, presented randomly according to an exponential distribution in order to prevent anticipatory responses. The task was to indicate whether the target appeared to the left (left key) or to the right (right key) of the fixation point (CRT task). The experiment involved 17 experimental sessions of 300 trials. Thus, 510 RTs were recorded for each subject and for each level of luminance. For each subject and each intensity, median RTs were calculated for each session and the medians were averaged over sessions.

\section{Results}

The overall mean difference between the RTs for the localization (left/right) of the stimulus, was $21 \mathrm{msec}$ $[F(1,3)=1.07$, n.s.]. For further analyses, the data were averaged over the localization factor, which had no significant effect.

Averaged over subjects, the percent of correct responses increased with increasing luminance. The data are illustrated in Figure 1A, using the probability of correct responses $\left(p_{\mathrm{CR}}\right)$ as ordinate. Clearly, two regions emerged from the data. In the upper range of intensities, the percentage of correct detections became statistically constant.

To provide a rough estimate of the upper limit of the threshold region, two linear regressions were carried out 

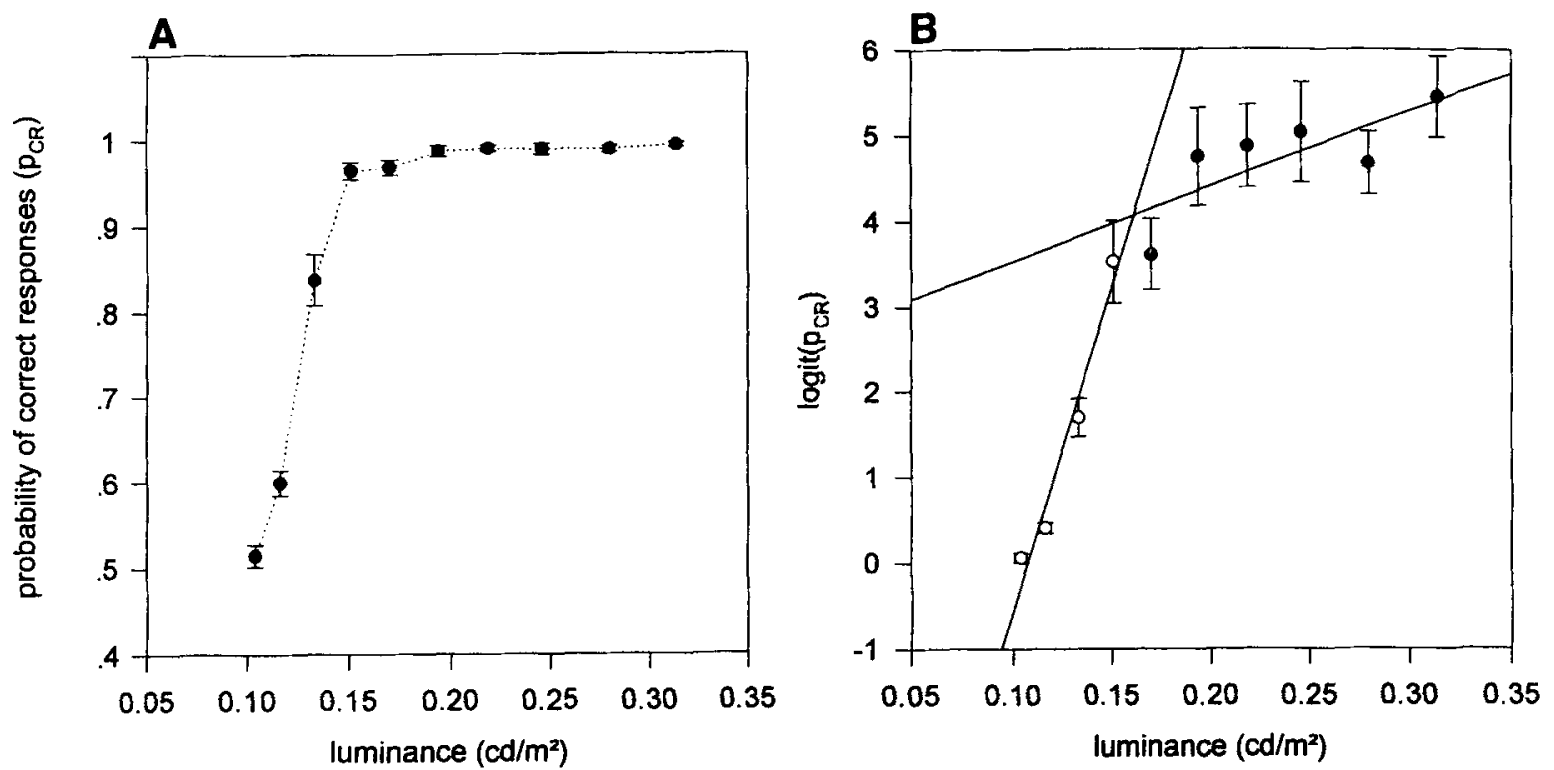

Figure 1. (A) Probability of correct responses as a function of the luminance level of the stimulus. (B) The probabilities that have been transformed in logits. The two linear functions giving the best fit are adjusted to these data (open symbols for the luminances in the threshold region). Bars are the interindividual standard errors of the means.

by iteration on the logit transforms of $p_{C R}[\operatorname{logit}(p)=$ $\ln [p /(1-p)]$. The psychometric function that relates the probability of correct detection to intensity (Bonnet, 1986) was fitted to the low range and another similar function to the upper range, and the $r$ values were used to test the goodness of fit. Complementarily, the fit of two functions was evaluated in calculating the sum of the squared residuals between the observed $\operatorname{logit}(p)$ and its theoretical value. This statistic $\left(\sum d^{2}\right)$, which is more sensitive than the correlation coefficient, was minimal for the best fit of the two functions (e.g., Pins \& Bonnet, 1997). The results are shown in Figure 1B. The best fit $\left(\sum d^{2}=136.5\right)$ was obtained for the lower range, for intensities between 0.104 and $0.151 \mathrm{~cd} / \mathrm{m}^{2}$ with the use of the following equation of the psychometric function:

$$
\operatorname{logit}(p)=70.25 \mathrm{~L}-7.5(r=.9844) .
$$

The detection threshold that corresponds to $75 \%$ correct responses is $\theta=0.122 \mathrm{~cd} / \mathrm{m}^{2}$. The individual estimates of the psychometric functions are similar as is shown in Table 1.

In the upper range of intensities $\left(>0.151 \mathrm{~cd} / \mathrm{m}^{2}\right)$, the statistical consistency between the subjects was lessened. There were at least two possible reasons for this. First, the probability of correct responses was not expected to change with luminance. Second, it was likely that every subject adopted a different criterion for the speed-accuracy tradeoff. The following equation fits the mean results:

$$
\operatorname{logit}(p)=8.34 \mathrm{~L}+2.41(r=.8164) .
$$

The mean RT were first analyzed independently of response types (correct or error). As is shown in Figure 2A, the RT decreased with increasing luminance of the stimuli. The mean RT difference between the two extreme levels of luminance was $223 \mathrm{msec}$. The between-subjects standard deviation was $23 \mathrm{msec}$. The Piéron function (Equation 1) fits the mean data over the entire range of luminances. The three parameters of the function were estimated with the use of a nonlinear method (MarquardtLevenberg algorithm) with SigmaPlot software. This was an iterative procedure on the raw data. That procedure stops when the residual sum of squares no longer decreases significantly (i.e., convergence criterion). The results of such an estimation would be similar to those obtained in using a $\log -\log$ transform of the data (cf. for instance Bonnet et al., 1999; Mansfield, 1973).

The estimated parameters were $\alpha=-2.85$ and $t_{0}=318.6 \mathrm{msec}$. In order to compare the fit of a single function with the fit of two functions, we calculated the sum of the squared residuals of the observed RT and the function $\left(\Sigma d^{2}\right)$ and used it as the criterion for the goodness of fit (e.g., Pins \& Bonnet, 1997). For the single func-

Table 1

Individual Psychometric Functions for the Threshold Range

\begin{tabular}{cccc}
\hline Subject & Psychometric Function & $r$ & $\theta$ (Expl) \\
\hline C.L. & $\operatorname{logit}(p)=65.7 \mathrm{~L}-6.8$ & .979 & 0.1202 \\
S.D. & $\operatorname{logit}(p)=63.5 \mathrm{~L}-6.7$ & .933 & 0.1237 \\
L.F. & $\operatorname{logit}(p)=62.2 \mathrm{~L}-6.7$ & .965 & 0.1251 \\
D.P. & $\operatorname{logit}(p)=77.1 \mathrm{~L}-8.3$ & .919 & 0.1218 \\
\hline
\end{tabular}



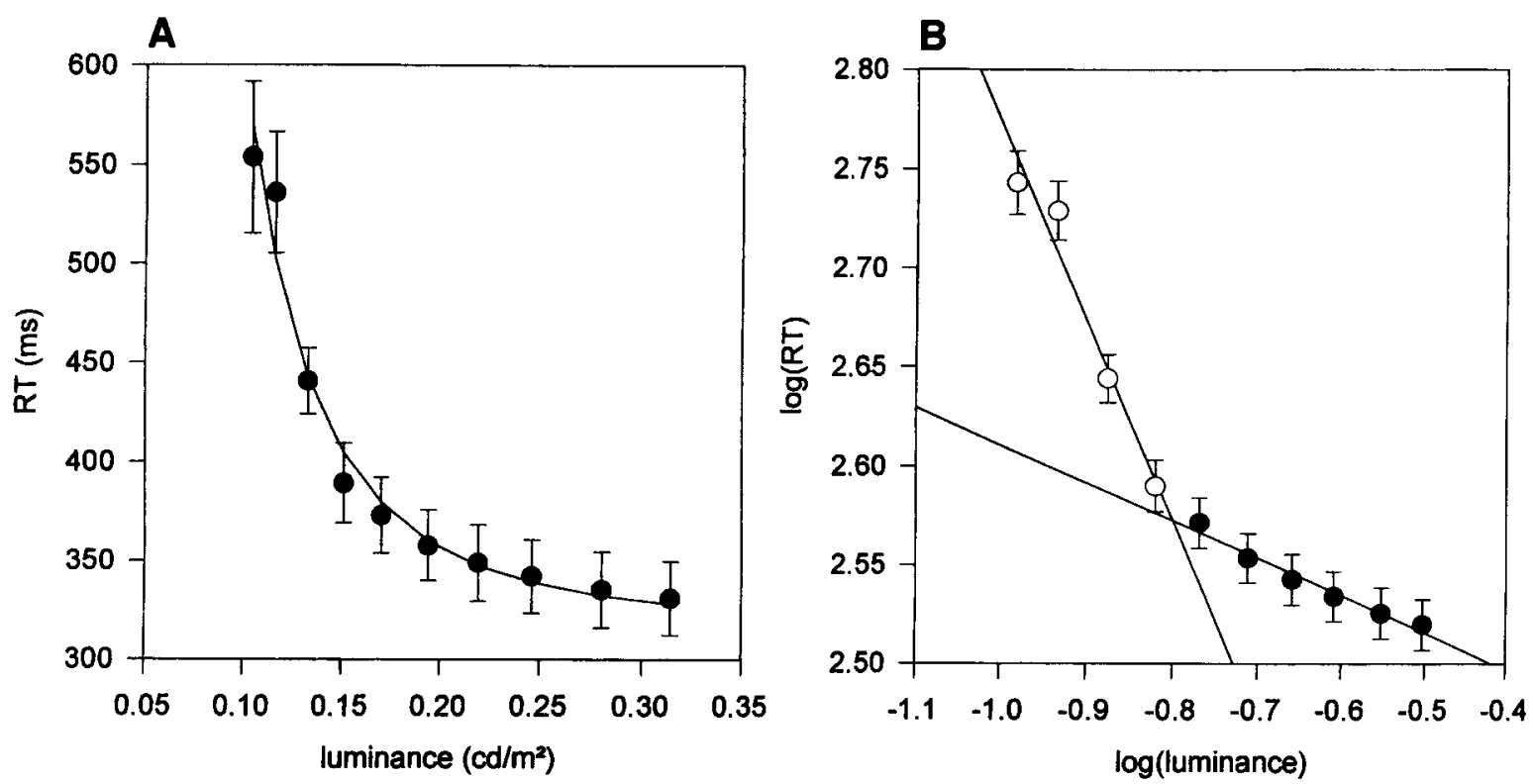

Figure 2. (A) RT as a function of luminance. Bars are the interindividual standard errors of the means. The Piéron function (Equation 1) was fitted to the data. (B) Two simplified Piéron functions (Equation 4) are fitted to the data shown here on a log-log plot. Open symbols for the data in the threshold region. Log standard errors have been divided by 100 for sake of clarity.

tion that fit the data with the parameters mentioned above, $\sum d^{2}=1,780.6$.

We then examined how two chronometric functions fit the results: one for the threshold range of luminance and one for the high range of luminance. The following simplified form of the Piéron function, which took into account only two parameters, was used:

$$
\mathrm{RT}=\beta I^{-\alpha} \text {. }
$$

The estimation of a third parameter $\left(t_{0}\right)$ would, in fact, not have been appropriate, especially for the lowest range of luminances. If Equation 1 (with three parameters) had been fit to the lower range of intensities, it would have led to a negative value of $t_{0}$ (precisely, $-1,000.6 \mathrm{msec}$ ) which would have been absurd. Using a two-parameter solution (Equation 4) for the entire range of luminances, while assuming the exponent is the same for all stimuli, produced a very poor fit $\left(\Sigma d^{2}=8,105.7\right)$. Then, two functions were fit to the results: one for the lower range and another for the upper range of intensities. This was accomplished by using the iterative procedure in which the limit between the lower and the upper ranges of luminances was changed at each run. The $\sum d^{2}$ summed over the two functions was used as a descriptive statistic for estimating the goodness of fit. The best fit was the one that minimized $\sum d^{2}\left(\sum d^{2}=\right.$

Table 2

Individual Exponents for the Two Ranges of Luminance

\begin{tabular}{ccc}
\hline Subject & Low Range & High Range \\
\hline C.L. & -1.56 & -0.22 \\
S.D. & -0.61 & -0.16 \\
L.F. & -0.39 & -0.18 \\
D.P. & -1.45 & -0.19 \\
\hline
\end{tabular}

963.5). This value was obtained with the same limit between the lower range and the upper range as the one found for the percent correct. This fit is illustrated in Figure 2B on a $\log -\log$ plot. The parameters of the two functions are

$$
0.104 \text { to } 0.151 \mathrm{~cd} / \mathrm{m}^{2}:-\alpha=-0.9793
$$

and

$$
0.170 \text { to } 0.314 \mathrm{~cd} / \mathrm{m}^{2}:-\alpha=-0.1959 \text {. }
$$

The same analysis was run subject by subject. It confirmed the hypothesis that using two separate functions holds individually. The exponents for the individual functions are shown in Table 2. Whereas the individual exponents are rather variable in the low range, the exponent of the function reduces drastically from the threshold range to the upper range of luminance for each subject. However, as mentioned above, the accuracy of the subject's performance was similar in the threshold range.

In a further analysis, the relationship between the accuracy and the RTs - that is, between the psychometric and the chronometric functions - was examined. As can be derived from Link's model (see, also, Bonnet, 1996; Link, 1992), a linear function with a negative slope is expected for the relationship between $\operatorname{logit}(p)$ and RTs. (The results are shown in Figure 6.) The following equation (Equation 5) fits the present data for the entire range:

$$
\mathrm{RT}=540.9-43.95 \operatorname{logit}(p)\left(\sum d^{2}=2,261.1\right) .
$$

However, as in the previous analysis, two regression functions provided a better fit $\left(\sum d^{2}=1,172.1\right)$. In the threshold region, the equation is

$$
\mathrm{RT}=550-52.4 \operatorname{logit}(p),
$$




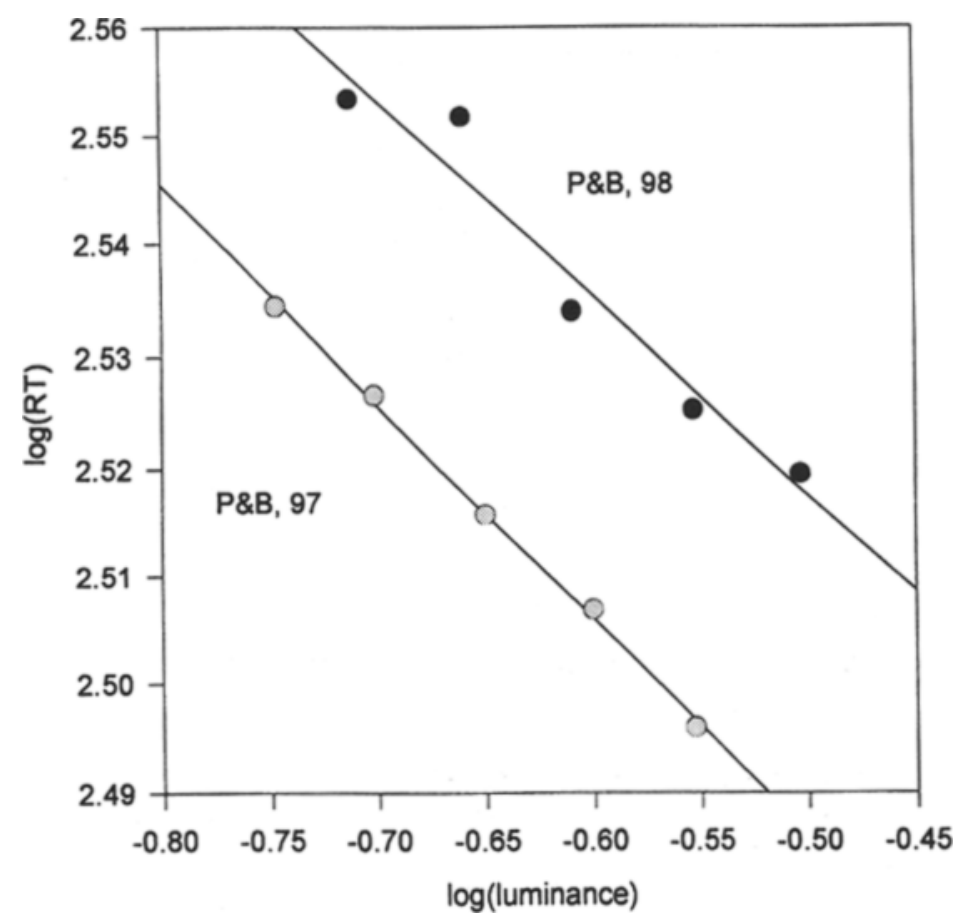

Figure 3. Comparison of the RT for the upper region of luminance between the data of the present experiment and those of Pins and Bonnet (1997).

whereas in the upper range, it is

$$
\mathrm{RT}=454.2-23.9 \operatorname{logit}(p) \text {. }
$$

In conclusion, the limit of the threshold region is equally well determined with an accuracy indice $[\operatorname{logit}(p)]$ and with the RT. The Piéron function that fit the data in this region had an exponent greater than the exponent found for the suprathreshold intensities.

Finally, to test the reliability of the present results in the upper range of intensities, we compared them with those obtained in a similar range in a previous experiment (Pins $\&$ Bonnet, 1997). The comparison is shown in Figure 3. On a $\log -\log$ plot, the results of the two experiments are very parallel, confirming the validity of the estimate of the exponent in the scotopic range of luminances. The difference between the two experiments was due to the fact that some of the subjects were not the same in the two experiments and were small in number. The "new" subjects were not as trained as the others and, as a consequence, were slower in responding.

\section{EXPERIMENT 2 \\ Choice Reaction Times in the Threshold Region}

The estimate of the threshold region may have been biased by the use of a relatively large range of luminances. This bias may have affected the psychometric function or the chronometric function, or both. Furthermore, only four luminance levels in Experiment 1 belonged to the threshold region. We conducted a second experiment with intensities in the threshold region only in order to collect additional data for both the psychometric and the chronometric functions. The luminance thresholds were measured with the method of constant stimuli in a forcedchoice situation.

\section{Method}

Subjects. Three trained subjects took part in the experiment. Two of the subjects, including the first author, took part in Experiment 1 . The subjects had normal or corrected-to-normal vision.

Procedure. The stimuli and experimental design were identical to those used in Experiment 1. However, in order to obtain six luminance levels centered around the detection threshold, an initial estimate of this threshold was first obtained using an adaptive method.

The detection threshold values can vary from subject to subject, and from session to session. Therefore, in order to determine the threshold region, each experimental session started with a preliminary estimate of the threshold. An adaptive procedure based on Levitt's (1971) was used. Two staircases, one ascending and one descending, were run and mixed randomly from trial to trial. The same spatial $2 \mathrm{AFC}$ method as in Experiment 1 was used. Within each series, the rule for changing the luminance value was that there had to be two successive trials with a correct response for a decrease of one step and one error for an increase. The threshold corresponds to $71 \%$ correct detections. Initially, the steps were about $0.015 \mathrm{~cd} / \mathrm{m}^{2}$ and then were reduced to $0.005 \mathrm{~cd} / \mathrm{m}^{2}$ after the first inversion of responses. The stopping criterion was based on the estimation of the slope for the last 10 trials as a function of their rank within each series. When the slope of each series was $0 \pm 0.01$, the experiment stopped and the averaged value of these 10 trials was taken as threshold.

Then, the six luminance values to be used in the main experiment were determined in a geometrical series with a ratio of 1.03 . The threshold that was measured with the adaptive method was the third 

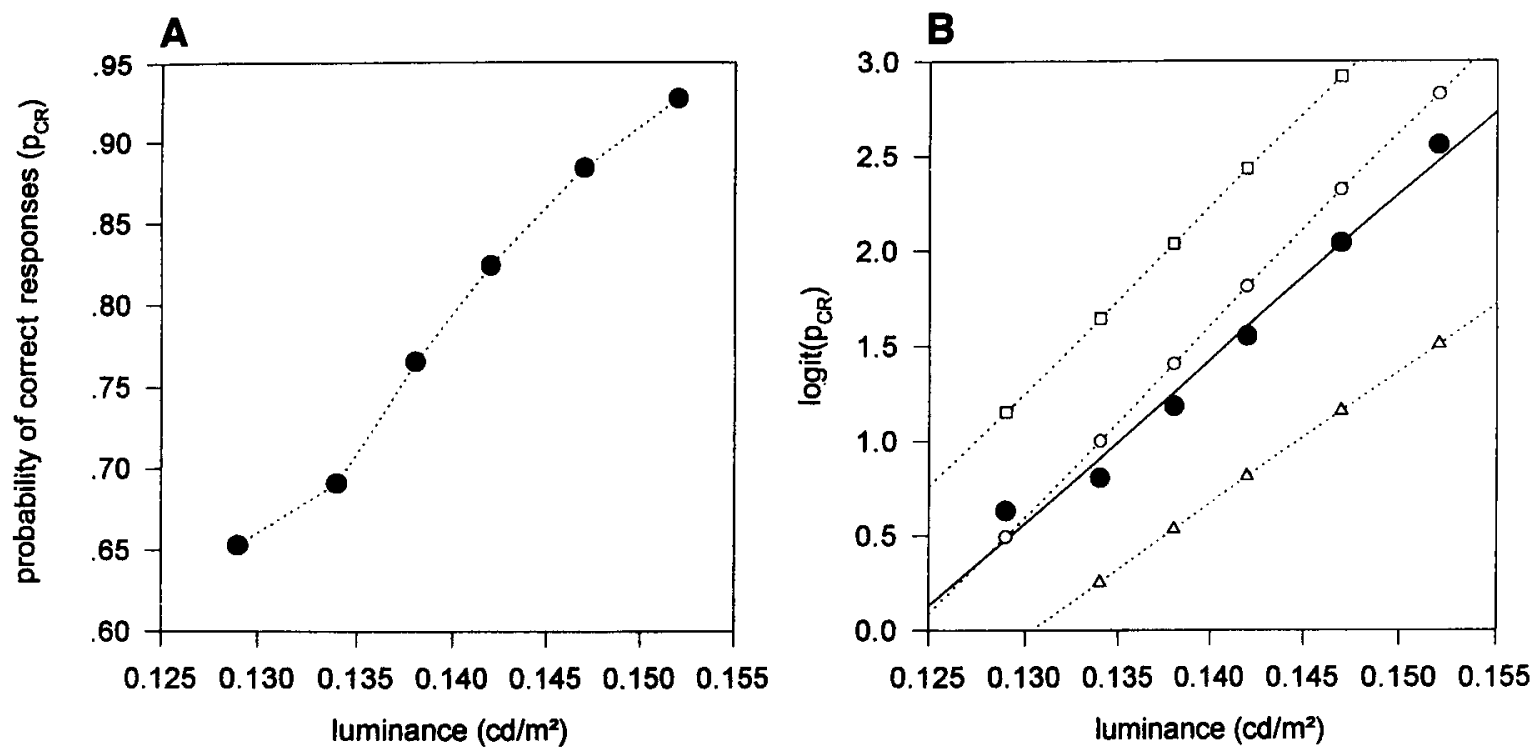

Figure 4. (A) Averaged probability of correct responses as a function of the luminance level of the stimulus for Experiment 2. (B) The probabilities have been transformed in logits. The linear function giving the best fit is adjusted to the data. Individual psychophysical functions are shown with open symbols.

intensity in the series. The six luminance levels were presented according to the constant stimuli method. The subjects went through 14 experimental sessions of 240 trials each. Thus, 560 RTs were recorded for each subject and for each level of luminance.

\section{Results}

The detection thresholds that were estimated with the adaptive method in the preliminary phase did not vary much from session to session. They ranged between 0.12 and $0.18 \mathrm{~cd} / \mathrm{m}^{2}$ for each subject with an average of $0.138 \mathrm{~cd} / \mathrm{m}^{2}$. Because the differences in luminances for each step of the main experiment were negligible from session to session and from subject to subject, the six following values were used in the next parametric analysis of the data: $0.129,0.134,0.138,0.142,0.147$, and $0.152 \mathrm{~cd} / \mathrm{m}^{2}$.

The RTs did not differ significantly $(F<1)$ for the two localizations (left/right) of the target. The averaged difference was $0.2 \mathrm{msec}$. For further analyses, the data were averaged over this factor.

The percentage of correct responses was transformed into logits and a psychometric function was computed (see Figure 4). The individual and mean results are shown in Table 3, which includes the comparison between the threshold estimated from the constant method [ $\theta$ (const)] and the thresholds estimated previously with the adaptive method [ $\theta$ (adapt)]. They both corresponded to $75 \%$ correct. The between-subjects standard deviation of mean percentage of correct responses was $1.5 \%$.

The mean thresholds that were estimated with the adaptive method were systematically larger than those obtained with the method of constant stimuli. The latter were, on the average, larger than those estimated from Experiment 1 $\left(\theta=0.122 \mathrm{~cd} / \mathrm{m}^{2}\right)$. Because the two extreme luminances in the range used in this second experiment were more distant from the minimum and the maximum of the probability function, the estimate of the threshold could be considered to be more reliable in this experiment. Nevertheless, the estimates of the upper limit of the threshold range were consistent in the two experiments.

As in Experiment 1, the RTs were first analyzed independently of response types (correct or errors). As is shown in Figure 5, CRT decreased with the increasing luminance of the stimuli. The mean RT difference between the two extreme levels of luminance was $77 \mathrm{msec}$. As in Experiment 1, a three parameter solution (Equation 1) produced a negative value of $t_{0}$, the goodness of fit being as good with a two-parameter solution (Equation 4). The estimated exponent of the Piéron function (Equation 4) that fit all the data $(r=-.99)$ is as follows:

$$
-\alpha=-1.0204 \text {. }
$$

Similar results were observed for each subject. Individual exponents of the Piéron functions were (DP) -1.16 , (SD) -0.91 , and (TP) -0.98 . The between-subjects standard deviation of mean RT was $21 \mathrm{msec}$. The analysis of the mean RTs only for correct responses, led to similar results. However, the mean RT for errors $(474 \mathrm{msec})$ was a little longer than the one for correct responses $(456 \mathrm{msec})$.

Table 3

Individual and Mean Psychometric Functions and Threshold Estimates

\begin{tabular}{lllccc}
\hline Subject & \multicolumn{2}{c}{ Psychometric Function } & $r$ & $\theta$ (const) & $\theta$ (adapt) \\
\hline D.P. & $\operatorname{logit}(p)=101.5 \mathrm{~L}-12.6$ & .989 & 0.135 & 0.141 \\
S.D. & $\operatorname{logit}(p)=98.1$ L -11.5 & .963 & 0.129 & 0.168 \\
T.P. & $\operatorname{logit}(p)=69.8$ L -9.1 & .992 & 0.146 & 0.180 \\
Mean & $\operatorname{logit}(p)=86.7$ L -10.7 & .991 & 0.136 & 0.161 \\
\hline
\end{tabular}




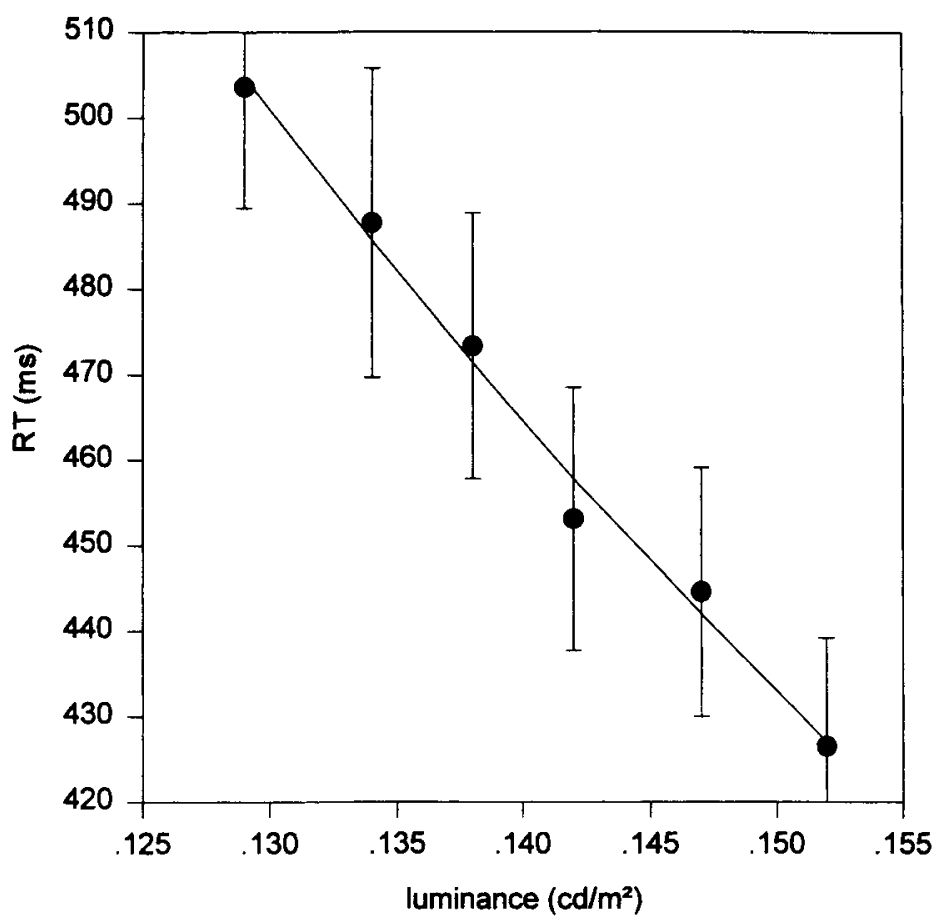

Figure 5. RT as a function of luminance. The Piéron function (Equation 2) was fitted to the data. Bars are the interindividual standard errors of the means.

The estimated exponent of the Piéron function on the mean RT for correct responses $(r=-.99)$ is $-\alpha=-0.9212$.

Finally, the RTs were plotted as a function of accuracy (see Figure 6B). As in Experiment 1, a linear relationship was demonstrated here between the RT and $\operatorname{logit}(p)$, with the following parameters:

$$
\mathrm{RT}=520.48-38.1 \operatorname{logit}(p)(r=.9995) .
$$

Figure 6 compares the results of the two experiments in the threshold region. The linear relationship is

$$
\mathrm{RT}=b-a \operatorname{logit}(p) \text {. }
$$

This fits the individual data and the averaged ones. However, although the mean threshold was similar in the two experiments, the slopes of the function (Equation 9) showed some individual differences (see Subject C.L. in Experiment 1, for instance). The average thresholds were a little lower in the first experiment than in the second one. The RT for the mean threshold values (obtained using Equation 9) were slightly longer in the first experiment than in the second one ( $496 \mathrm{vs} .475 \mathrm{msec}$ ). The tradeoff between accuracy (threshold) and speed could result from a "context" effect. In Experiment 2, the conditions were more consistent because all levels of luminance belonged to the threshold region. In Experiment 1, only 4 out of 10 belonged to that region. The fact that the majority of the luminance levels were supraliminal may have encouraged the subjects to respond faster. Such an interpretation is reinforced by the fact that there were errors even when the luminance was clearly a suprathreshold one.

\section{Discussion}

The two experiments confirmed that the Piéron function holds both in the threshold range of luminances and above that range. However, the exponent of the function increased drastically in the threshold region. In fact, not only did the RT decrease with the increase in luminance, it also did so when the probability of correct detection increased. As a result of the combined effect of these two factors, a linear relationship is demonstrated between the logit transform of detection probability and the RT (Equation 9). The Piéron functions in the two present experiments that fit the threshold range, are parametrically very similar with an exponent close to 1 (Experiment $1,-\alpha=$ -0.9793; Experiment 2, $-\alpha=-1.0204$ ).

As was shown in Experiment 1, the exponent of the function decreases drastically for stimulus luminance beyond the limit of the threshold region. This result confirms observations on Piéron's (1914) or Chocholle's (1940) data. When working with the Piéron function, it is therefore critical to determine the threshold region in order to avoid bias in the estimation of the exponent. Empirically, it would seem convenient to look for the weight of the lowest intensities (see Bonnet et al., 1999).

The increase in the exponent for the lower range of intensities (the threshold region) demonstrated by our RT 

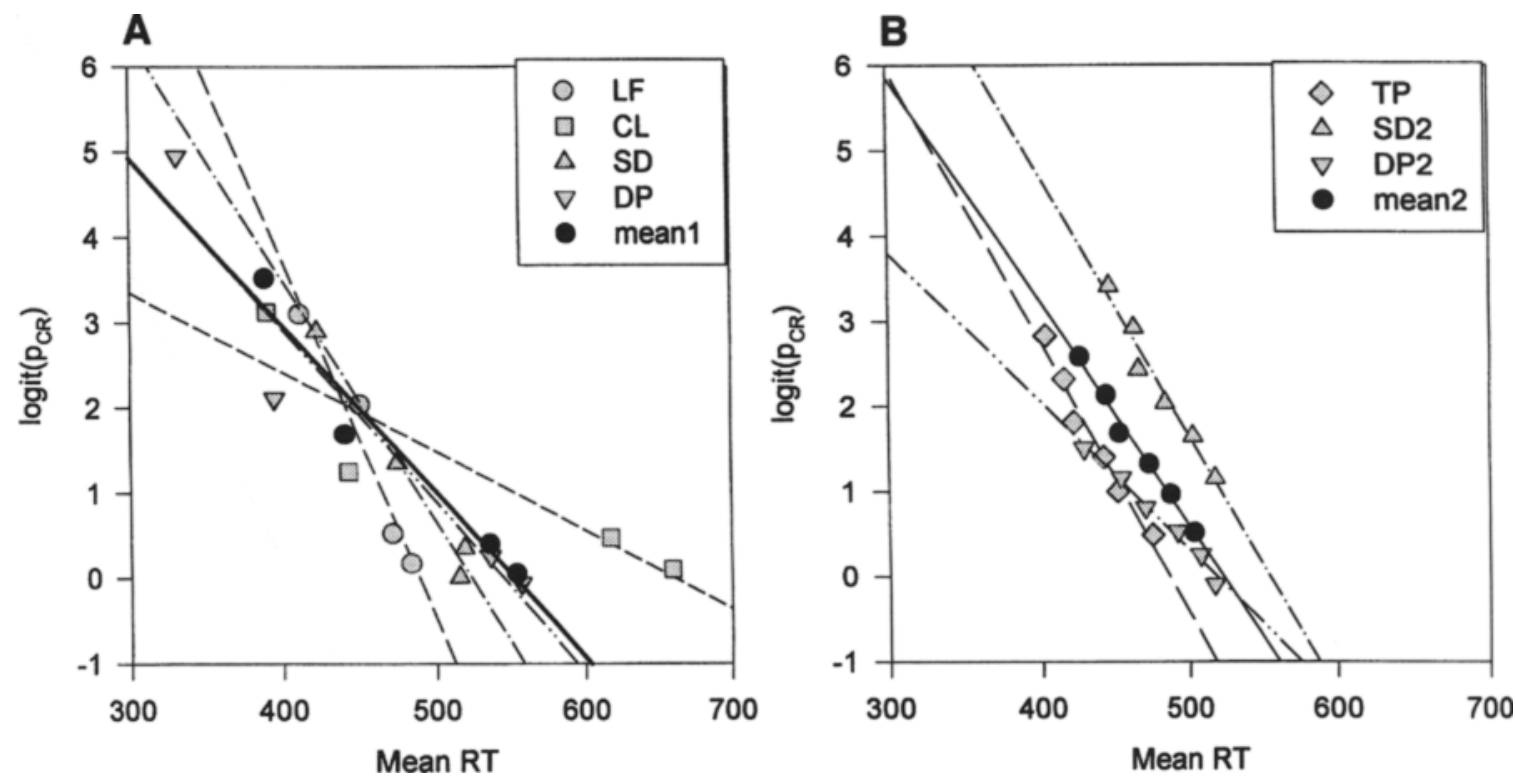

Figure 6. Linear relationship between RT and $\operatorname{logit}\left(p_{\mathrm{CR}}\right)$ in Experiment $1(\mathrm{~A})$ and in Experiment 2 (B). In dark are the averaged data, in gray are the individual results.

data is parallel to the increase in the exponent of the Stevens's function applied to magnitude estimation data (ME), as examined previously by Baird (1997). There are theoretical arguments (Link, 1992; Norwich, 1993) that the absolute value of the exponents of the Pieron and the Stevens functions should be the same. However, no direct comparison of these exponents can be made here. Magni- tude estimation data would have to be obtained under the same experimental conditions.

Different explanations can be brought forward to explain the steeper exponent in the threshold region, such as, for example, when intensity increases near threshold, uncertainty will decrease. At low intensities, only a relatively small number of neurons, those having a low dy-
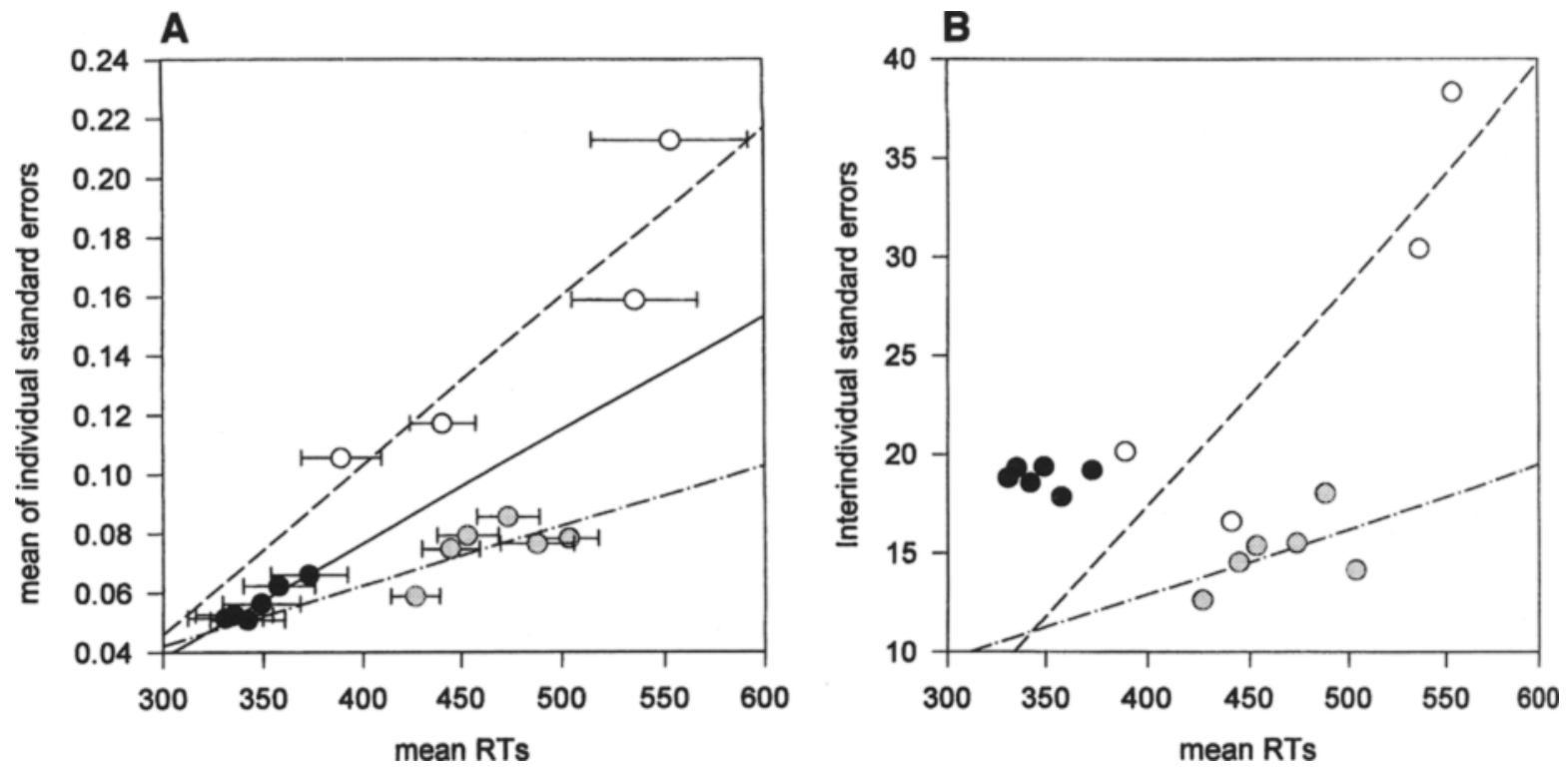

Figure 7. Relationships between the mean RTs and variability. Results are shown on a $\log -\log$ plot for more clarity. (A) The ordinate is the mean of the individual standard errors. (B) The ordinate is the interindividual standard error of the mean. Open symbols are results of Experiment 1 in the threshold region, black symbols are the results of Experiment 1 above the threshold region, gray symbols are the results of Experiment 2 in the threshold region. 
namic range and, as a consequence, having a low threshold (Baird, 1997) would be activated. These neurons, being hypothetically more sparse, would reduce the probability of an early response and therefore lengthen the RT. However, this explanation is not sufficient to explain why the RT still declines beyond the threshold region. Increasing the intensity of the stimulus increases the speed of summation of receptor potentials and thus reduces the delay between receptor activation and neural action potentials. It also increases neural firing rates. All of these factors operate on a large range of intensities beyond threshold. As far as the RT is concerned, a saturation point is reached when the RTs become constant $\left(t_{0}\right)$, and the Piéron function and the Stevens function should diverge.

A complementary line of thought relates the Piéron or Stevens functions to discrimination (Link, 1992; Norwich, 1993; Teghtsoonian, 1971). Whereas the present experiments do not relate directly to that issue, an analysis of changes in the variability of RTs provides an argument in favor of this idea. Analyses of the RT revealed both interand intrasubject variabilities. Baird (1997) mentioned that some studies appear to be at odds with each other regarding the relationship between the mean RT and its variability. On the one hand, Chocholle (1940) showed a linear relationship between mean RT and the standard deviation, which is consistent with Weber's law. That linear relationship has been stressed by Bonnet (1996), and has been found to apply to various sets of data. On the other hand, Green and Luce (1971) have shown data exhibiting a nonlinear function that is concave downward. In Experiment 1 and in Experiment 2, we examined the relationship between the individual mean RT and the standard errors. For each subject in each experiment, a linear regression fit satisfactorily to these data (see Figure 7). A nonlinear function (power function) was investigated, and it was found that it did not improve the goodness of fit substantially. However, in each case the function appeared slightly concave upward rather than concave downward. In Experiment 1, nonlinearity seems to have resulted mainly from the separation between the threshold range and the suprathreshold range. A similar analysis was performed on interindividual variability, leading to similar conclusions. These data are presented in a condensed way in Figure 7. To the best of our knowledge, they do not allow one to draw a conclusion about this issue, essentially because there were a limited number of observations.

The asymptotic RT, $t_{0}$, may also deserve some further comments. In Piéron's data on RT and brightness (1914), $t_{0}$ was estimated within the range of $100-150 \mathrm{msec}$ in Mansfield (1973) the best estimate was $194.6 \mathrm{msec}$. Apart from the numerous problems in getting a stable estimate of this parameter (Bonnet et al., 1999; Luce, 1986), its value depends on several factors. The first factor relates to the task itself. The task here was a CRT task, whereas previously mentioned results were obtained in SRT tasks. A difference in the parameter $t_{0}$ in these two tasks has been shown by Pins and Bonnet (1996). A second set of factors, discussed by Luce (1986), relates to interindividual differences and to the degree of training of the observers.

In CRT tasks, errors are not always related to the detectability of the stimuli. In our Experiment 1, for example, some errors were observed even when the luminance levels were beyond the estimated limit of the threshold region. These errors may have resulted from the time pressure induced by the experiments. However, as seen previously (Pins \& Bonnet, 1996, 1997), they do not, in general, vary with luminance beyond the threshold region.

In conclusion, we have demonstrated in our experiments that uncertainty about the presence of the stimulus in the threshold range makes the Piéron function steeper than beyond that range. We have contributed to reinserting RT measures into the field of sensory psychophysics, where they have been somewhat neglected in the past. From our discussion, there appeared to emerge many questions that call for further investigation in this domain.

\section{REFERENCES}

BAIRD, J. C. (1997). Sensation and judgment: Complementarity theory of psychophysics. Mahwah. NJ: Erlbaum.

BARTLETT, J. R. \& DOTY, R. W. (1974). Response of units in striate cortex of squirrel monkeys to visual and electrical stimuli. Journat of Neurophysiology, 37, 621-641.

Bonnet, C. (1986). Manuel Pratique de Psychophysique. Paris: Armand Colin.

BonNeT, C. (1992a). Psychophysical scaling within an information processing approach? Behavioral \& Brain Sciences, 15, 560-561.

BONNET. C. (1992b). Sensation and psychophysics of reaction time. International Journal of Psychology, 27, 373.

BONNET. C. (1996). Sensory/decisional problem: An expansion of Link's theory. In S. C. Masin (Ed.), Fechner Day 96 (pp. 137-142). International Society for Psychophysics.

Bonnet. C.. Zamora, M. C.. Buratti, F. \& Guirao. M. (1999). Group and individual gustatory reaction times and Piéron's law. Physiology \& Behavior, 46, 1-10.

Bujas. Z. (1935). Le temps de réaction aux excitations gustatives d'intensité différente. Comptes Rendus de la Société de Biologie, 119. $1360-1364$

CAtTell.J. M. (1886). The influence of the intensity of the stimulus on the length of the reaction time. Brain, 8, 512-515.

Chocholle, R. (1940). Variation des temps de réaction auditifs en fonction de l'intensité à diverses fréquences. L'Année Psychologique, 41-42, 65-124.

ExNER, S. (1868). Über die zu einer Gesichtswahrnehmung nötige Zeit. Sitzungsberichte der Kaiserlichen Akademie der Wissenschaften, 57, 601-632.

GREen, D. M., \& LUCE, R. D. (1971). Detection of auditory signals presented at random times: III. Perception \& Psychophysics, 9, 257-268.

Grice, G. R. (1968). Stimulus intensity and response evocation. Psychological Review, 75, 359-373.

HARA, S. (1955). Interrelationships between stimulus intensity, stimulated areas and reaction times in human gustatory sensation. Bulletin of Tokvo Medical \& Dentistry (Iniversity, 2, 147-158.

LAPPIN, J. S.. \& DisCH. K. (1972). The latency operating characteristic: II. Effects of visual stimulus intensity on choice reaction time. Journal of Experimental Psychology, 93, 367-372.

LEVICK, W. R. (1973). Variation in the response latency of cat retinal ganglion cells. Vision Research, 13, 837-853.

LEvitT, H. (1971). Transformed up down methods in psychoacoustics. Journal of the Acoustical Society of America, 49, 467-477.

Link, S. W. (1978). The relative judgment theory of the psychometric 
function. In J. Requin (Ed.), Attention and performance VII (pp. 619630). Hillsdale, NJ: Erlbaum.

LiNK, S. W. (1992). The wave theory of discrimination and similarity. Hillsdale, NJ: Erlbaum.

LuCE, R. D. (1986). Response times. New York: Oxford University Press.

LUCE, R. D., \& GREEN, D. M. (1972). A neural timing theory for response times and the psychophysics of intensity. Psychological Review, 79, 14-57.

MANSFiELD, R. J. W. (1973). Latency functions in human vision. Vision Research, 13, 2219-2234.

Marrocco, R. T. (1975). Possible neural basis of brightness magnitude estimations. Brain Research, 86, 128-133.

McGil., W. J. (1961). Loudness and reaction time: A guided tour of the listener's private world. Acta Psychologica, 19, 193-199.

McGILL, W. J. (1963). Stochastic latency mechanisms. In R. D. Luce, R. R. Bush, \& E. Galanter (Eds.), Handbook of Mathematical Psychology (pp. 309-360). New York: Wiley.

NoRwich, K. H. (1993). Information, sensation and perception. San Diego, Academic Press.

Pachella, R. G., \& Fisher, D. F. (1969). Effect of stimulus degradation and similarity on the trade off between speed and accuracy in absolute judgments. Journal of Experimental Psychology, 81, 7-9.

Piéron, H. (1914). Recherches sur les lois de variation des temps de latence sensorielle en fonction des intensités excitatrices. L'Année Psychologique, 20, 17-96.

Piéron, H. (1920). Nouvelles recherches sur l'analyse du temps de latence sensorielle en fonction des intensités excitatrices et sur la loi qui relie ce temps a l'intensité de l'excitation. L'Année Psychologique, 22, 58-142.

PiÉRon, H. (1952). The sensations. New Haven, CT: Yale University Press.

PINS, D., \& BonNeT, C. (1996). On the relation between stimulus intensity and processing time: Piéron's law and choice reaction time. Perception \& Psychophysics, 58, 390-400.
Pins, D., \& Bonnet, C. (1997). Reaction times reveal the contribution of the different receptor components in luminance perception. Psychonomic Bulletin \& Review, 4, 359-366.

POSNER, M. I. (1986). Chronometric exploration of mind. Oxford: Oxford University Press.

RESTLE, F. (1961). The psychology of judgment and choice: A theoretical essay. New York: Wiley.

SChWeickert, R., DAhN, C.. \& MCGuigan, K. (1988). Intensity and the number of alternatives in hue identification: Piéron's Law and choice reaction time. Perception \& Psychophysics, 44, 383-389.

Stevens. S. S. (1975). Psychophysics: Introduction to its perceptual. neural and social prospects. New York: Wiley.

Stone, J., \& Fukuda, Y. (1974). Properties of cat retinal ganglion cells: A comparison of W-cells with X-and Y-cells. Journal of Neurophysiology, 37, 722-748.

Teghtsoonian, R. (1971). On the exponents of Stevens' law and the constant of Ekman's law. Psychological Bulletin, 78, $71-80$.

Tepas, D. I.. \& ARmington, J. C. (1962). Properties of evoked visual potentials. Vision Research, 2, 449-461.

WOODWORTH, R., \& SCHLOSBERG, H. (1955), Experimental psychology. New York: Holt.

WundT, W. (1874). Grundzüge der Physiologisches Psychologie. Leipzig: Engelmann.

Yamamoto, T., Kato, T., Matsuo, R., Araie, N., Azuma, S., \& KawaMURA, Y. (1982). Gustatory reaction time under variable stimulus parameters in human adults. Physiology \& Behavior, 29, 79-84.

Yamamoto, T., \& Kawamura, Y. (1981). Gustatory reaction time in human adults. Physiology \& Behavior, 26, 715-719.

(Manuscript received April 8, 1998; revision accepted for publication November 19, 1998.) 